\title{
Bioluminescence Imaging to Study Mature Biofilm Formation by Candida spp. and Antifungal Activity In Vitro and In Vivo
}

\author{
Katrien Van Dyck 1,2 \\ Patrick Van Dijck $1 \bowtie, 2$ \\ Email: patrick.vandijck@kuleuven.vib.be \\ Greetje Vande Velde 3 \\ VIB-KU Leuven Center for Microbiology, Leuven, Belgium \\ 2 Laboratory of Molecular Cell Biology, Institute of Botany and Microbiology, KU Leuven, Leuven, Belgium \\ Biomedical MRI/MoSAIC, Department of Imaging and Pathology, KU Leuven, Leuven, Belgium
}

\section{Abstract}

The widespread use of indwelling medical devices has increased the number of device-related infections in hospitalized patients. These infections are often associated with the formation of biofilms on the medical implants that are difficult to treat because of their resistance to the classical antifungal drugs. The most common fungi isolated from catheters and other medical devices are Candida species. The Candida genus contains multiple species of which C. albicans and C. glabrata are the two most common pathogenic yeasts in humans. A limited number of animal models is available for investigating host-pathogen interactions and testing novel antifungal drugs in vivo against these species. Fungal load in biofilms in these models is traditionally analyzed postmortem, requiring host sacrifice and enumeration of microorganisms from individual biofilms in order to evaluate the amount of colony forming units and the efficacy of antifungal treatment. Bioluminescence imaging (BLI) made compatible with small animal models for in vivo biofilm formation is a valuable tool to follow biofilm development and its treatment longitudinally. Due to the noninvasive nature of BLI, the imaging procedure can be repeated in the same animal, allowing for follow-up of the biofilm growth in vivo without removing the implanted device or detaching the biofilm from its substrate. Although detecting a quantifiable in vivo BLI signal from biofilms formed on the inside of implanted catheters is challenging, BLI proved to be a practical tool in the study of fungal biofilms. This method describes the use of BLI for in vitro and in vivo follow-up of device-related fungal biofilm formation in mice and rats and antifungal activity testing against both C. albicans and C. glabrata device-associated biofilms. It can further be applied for efficient in vivo screening for interesting genes of the pathogen and the host involved in biofilm formation.

AQ1

\section{Key words}

\section{Candida albicans}

Candida glabrata

Biofilm

Bioluminescence imaging

Gaussia luciferase

Firefly luciferase

Coelenterazine

Luciferin

In vivo

Noninvasive

\section{Introduction}

\section{AQ2}

The emergence of fungal infections pose an increasing threat to human health. They have become a challenge in clinical practice due to the increasing antimicrobial resistance and the limited amount of available antifungal drugs [1]. Both yeasts (e.g.,

Cryptococcus neoformans) and molds (e.g., Aspergillus fumigatus) can cause human fungal infections but the majority is caused by the opportunistic pathogenic yeasts of the Candida genus $[2,3]$. These infections can be superficial and relatively harmless or systemic and life threatening, mainly in immunocompromised patients [4]. C. albicans and C. glabrata are respectively the first and second most common cause of invasive Candida infections in the USA and Europe [5]. Increasing numbers of fungal diseases caused by Candida species and other fungi are related to the increasing number of severely ill or immunocompromised patients and in particular to the growing use of implanted medical devices in patients $[2,6,7]$. 
and their characteristics have been mostly elucidated in vitro [9]. Although they give valuable information, these models cannot account for numerous host and infection-site variables that are important during the development of biofilm-related infection in humans. Therefore, in vivo central venous catheter (CVC) C. albicans biofilm models were introduced in rats [10], rabbits [11], and mice [12]. These models are very useful and relevant, but at the same time technically demanding and of very low throughput. To overcome this, a rat subcutaneous foreign body infection model has been optimized to study C. albicans biofilms $[13,14]$. Still, a limitation to all these models is that the fungal load is traditionally analyzed post mortem by enumeration of the number of colony forming units attached to the catheter fragments and from individual host organs to determine the extent and kinetics of dissemination during infection. Only one time point per animal can be measured and therefore large groups of animals are needed. These end-point assays do not always provide a full understanding of the dynamic steps in Candida biofilm development, nor account for interindividual variation.

Noninvasive imaging has the potential to deliver data over the biologically relevant time scale from individual animals in vivo, obviating the need to sacrifice animals at different stages of biofilm development. In recent years, bioluminescence imaging (BLI) has emerged as a powerful method to analyze infectious diseases in animal models and microbial viability in particular [15]. It has become the method of choice to detect fungal presence in living tissues because of the high signal-to-noise ratio [16]. There are several light production systems available in fungi of which two are discussed here: the Gaussia princeps luciferase (gLUC) and firefly luciferase (fLUC). A synthetic C. albicans codon-optimized version of the gene for the naturally secreted gLUC was designed and tagged to the C. albicans PGA59 gene, which encodes a glycosylphosphatidylinositol (GPI)-linked cell wall protein [17]. Therefore, the luciferase enzyme will be present extracellularly, which avoids potential problems with intracellular delivery of the substrate coelenterazine (CTZ). This expression system has made BLI of superficial C. albicans infections possible [17]. In contrast, the fLUC, which uses luciferin as a substrate, is expressed intracellularly because it needs ATP as a cofactor for light emission [18]. Recently, the fLUC was adapted to improve its thermostability and maximum emission intensity in the red light range for expression in Candida cells [16]. Red-shifted light of fLUC has the advantage of being less absorbed by host tissues in contrast to the blue light emitted by gLUC that has more limited tissue penetration. BLI of both superficial and systemic infections is possible with fLUC [19]. In addition, BLI is used to screen the antifungal activity of existing or potentially new antifungal drugs against device-associated Candida biofilms. The use of BLI reduces the high effort and ethical burden often associated with these studies and increases the throughput and efficiency, thereby refining preclinical therapy studies $[20,21]$.

Here we describe a multitemporal noninvasive imaging assay to follow mature biofilm formation and screen the efficacy of antifungals against biofilms in vitro and under in vivo conditions in mice and rats, avoiding the need to sacrifice the animals, which results in statistical, ethical, economic and experimental advantages. The use of in vivo imaging reduces the time, number of animals, and costs for the analysis of biofilm models and increases the versatility of the current animal models to the use of different transgenic mouse strains. The imaging protocol described here will be readily translatable for use with other in vivo biofilm models such as the CVC model, for the assessment of antifungal therapies and the validation in transgenic models to study microbial and host factors influencing biofilm formation [20,21, 22].

\section{Materials}

\subsection{Candida Strains}

1. C. albicans SC5314 (wild-type), a clinical isolate [23]. The genome of this clinical isolate was successfully sequenced.

2. C. albicans SKCA23 strain (named SKCA23-ACTgLUC) expressing gLUC fused to the endogenous PGA59 gene under the control of ACT1 (actin) promoter (this promoter is active in the yeast as well as hyphal stage of fungal growth). To construct this strain, C. albicans SC5314 was transformed with the Clp10::ACT1p-gLUC59 plasmid [17], which was integrated in the RPS10 locus, in order to constitutively express the codon-optimized Gaussia princeps luciferase gene (gLUC) at the cell wall.

3. C. glabrata ATCC2001 (wild-type), used to generate a histidine auxotrophic mutant. The HIS1 locus was deleted by transforming the wild-type with the BamHI restricted pUC19 plasmid containing an upstream and downstream fragment of the HIS1 locus and a nourseothricin resistance cassette [20].

4. C. glabrata ATCC2001 fLUC. The histidine auxotrophic C. glabrata was used to generate a bioluminescent strain with an optimized synthetic fLUC gene adapted to the codon usage of C. glabrata. The BamHI restricted pUC19 plasmid, containing the promotor of the enolase gene (pENO1) with the luciferase gene and homologous regions of the HIS1 locus for integration, was used to transform C. glabrata with histidine prototrophy as selection marker [20].

\subsection{Cell Culture and Media}

1. Standard cell culture medium: RPMI-1640, with L-glutamine and without sodium carbonate buffered with MOPS, $\mathrm{pH} 7.0$.

2. Phosphate buffered saline: for 11 of $10 \times \mathrm{PBS}$, dissolve $80 \mathrm{~g} \mathrm{NaCl}, 2 \mathrm{~g} \mathrm{KCl}, 14.4 \mathrm{~g} \mathrm{Na}_{2} \mathrm{HPO}_{4}, 2.4 \mathrm{~g} \mathrm{KH}_{2} \mathrm{PO}_{4}$ in sterile water. 
3. Sterile water for preparation of media.

4. YPD agar plates: $1 \%$ yeast extract, $2 \%$ Bacto peptone, $15 \%$ agar, supplemented with $2 \%$ glucose in sterile water.

5. Fetal bovine serum (FBS) for incubation of catheter pieces.

6. Cell culture plates: 96-well and 24-well black (or white, see Note 8) polystyrene plates.

7. Catheters: polyurethane (see Note 1) triple-lumen intravenous (i.v.) catheters (2.4 mm diameter, Certofix Trio S730, BBraun, see Note 2), the triple-lumen part cut into $1 \mathrm{~cm}$ long fragments.

8. Incubator at $37^{\circ} \mathrm{C}$ for static incubation of YPD plates.

\subsection{Animals}

Before planning animal experiments, seek approval from your local bioethics committee and follow the respective guidelines for animal handling. Always take care that you carry out all aspects of animal experiments in compliance with national and supranational regulations regarding animal handling and welfare.

1. Mice: female Balb/C mice 8 weeks of age, kept in individually ventilated filter top cages with free access to standard food and water ad libitum (see Note 3 ).

2. Rats: Sprague-Dawley female rats of $200 \mathrm{~g}$ kept in individually ventilated filter top cages with free access to standard food and water ad libitum.

3. Suppress the immune system of the rats (optional in mice: see Note 4) $24 \mathrm{~h}$ before catheter implant and continue during the entire experiment by adding dexamethasone $(0.4 \mathrm{mg} / \mathrm{l})$ to their drinking water.

4. When using immune suppression, also add antibiotics (e.g., ampicillin sodium powder $0.5 \mathrm{~g} / \mathrm{l}$ ) to the drinking water to avoid possible bacterial infections.

\subsection{Anesthesia and Surgery}

1. Anesthesia for mice: prepare $1 \mathrm{ml}$ of anesthetic cocktail by mixing $75 \mu$ l of ketamine (Ketamine $\left.1000^{\circledR}, 100 \mathrm{mg} / \mathrm{ml}\right)$ with $100 \mu \mathrm{l}$ of medetomidine (Domitor ${ }^{\circledR}, 1 \mathrm{mg} / \mathrm{ml}$ ) and $825 \mu \mathrm{l}$ of sterile saline. Administer intraperitoneally (i.p.) $60-80 \mu \mathrm{l} \mathrm{of}$ anesthetic cocktail per $10 \mathrm{~g}$ body weight, resulting in a dose of $45-60 \mathrm{mg} / \mathrm{kg}$ ketamine and $0.6-0.8 \mathrm{mg} / \mathrm{kg}$ medetomidine. For reversal of anesthesia, dilute $50 \mu \mathrm{l}$ atipamezole (Antisedan ${ }^{\circledR}, 5 \mathrm{mg} / \mathrm{ml}$ ) in $4.95 \mathrm{ml}$ saline, administer i.p. $100 \mu \mathrm{l}$ per $10 \mathrm{~g}$ body weight as antidote, resulting in a dose of $0.5 \mathrm{mg} / \mathrm{kg}$.

2. Anesthesia for rats: prepare $1 \mathrm{ml}$ of anesthetic cocktail by mixing $600 \mu \mathrm{l}$ of ketamine with $400 \mu \mathrm{l}$ of medetomidine. Administer i.p. $100 \mu \mathrm{l}$ of anesthetic cocktail per $100 \mathrm{~g}$ body weight, resulting in a dose of $60 \mathrm{mg} / \mathrm{kg} \mathrm{ketamine}$ and $0.4 \mathrm{mg} / \mathrm{kg}$ medetomidine. For reversal of anesthesia, dilute $1 \mathrm{ml}$ atipamezole in $4 \mathrm{ml}$ saline; administer i.p. $100 \mu \mathrm{l}$ per $100 \mathrm{~g}$ body weight as antidote, resulting in a dose of $1 \mathrm{mg} / \mathrm{kg}$.

3. Xylocaine gel $(2 \%)$ as local anesthetic for the skin.

4. Terramycin/polymyxin-b ophthalmic ointment to apply on the eyes to prevent them from drying/infecting while the animal is under anesthesia.

5. Electric trimmer/razor or disposable razors for shaving the back of the animals.

6. Clean, sterile surgical tools: fine scissors, tweezers, surgical thread, needle and tools for suturing the skin.

7. Sterile gauze, iodoisopropanol (1\%) as a disinfectant for the skin.

8. Heating plate or mat to keep the animals warm while they are under anesthesia.

9. Extra clean cages kept warm (on a warm plate or with a lamp) to keep animals separate until they are fully recovered from anesthesia and surgery. 
1. BLI camera: We have used IVIS 100 and IVIS Spectrum systems (Perkin Elmer, USA) and Living Image software (version 2.50.1 and 4.5.2 for PC, provided by the manufacturer) for measuring and quantifying luminescence in vitro (see Note 5) and in vivo (see Note 6).

2. Coelenterazine solution: native coelenterazine (CTZ) was stored at $-80{ }^{\circ} \mathrm{C}$ according to the manufacturer's instructions. $5 \mathrm{mg} / \mathrm{ml} \mathrm{CTZ}$ were dissolved in acidified ethanol according to the manufacturer's instructions. This stock solution can be stored at $-80^{\circ} \mathrm{C}$ for the duration of the experiments, typically 6-9 days. For in vitro BLI, $6 \mu \mathrm{M}$ CTZ working solution was prepared by diluting the stock solution 1:2000 in sterile PBS. For in vivo BLI, we prepared a $1.2 \mathrm{mM}$ working solution by diluting the stock solution 1:10 in sterile PBS (see Note 7). Prepare $100 \mu \mathrm{l} \mathrm{CTZ} \mathrm{working} \mathrm{solution} \mathrm{per}$ catheter trio. Insulin syringes $(0.3 \mathrm{ml})$ were used for injections. CTZ is light-sensitive, always keep it in the dark (e.g., cover recipients with aluminum foil).

3. D-Luciferin solution: For in vitro and in vivo BLI, D-luciferin sodium salt 99\% (BC218, Synchem, Germany) was dissolved in sterile saline $(0.9 \% \mathrm{NaCl})$ at a concentration of $33.3 \mathrm{mg} / \mathrm{ml}$ (stock solution). For in vitro experiments, the Dluciferin stock solution was further diluted in sterile saline to $100 \mathrm{mg} / \mathrm{ml}$. For in vivo BLI, $100 \mu \mathrm{l}$ of the D-luciferin stock solution is needed per injection site. Insulin syringes $(0.3 \mathrm{ml})$ were used for injections. D-Luciferin is light-sensitive, always keep it in the dark (e.g., cover recipients with aluminum foil).

\section{Methods}

Follow national regulations for handling microbial pathogens class 2. Perform all handlings with open C. albicans suspensions in a vertical laminar airflow cabinet (LAF cabinet) to avoid contamination of cell cultures.

\subsection{Bioluminescence Imaging of In Vitro Biofilms}

\subsubsection{In Vitro Biofilm Formation}

1. Grow the C. albicans or C. glabrata strains you want to include in your experiment (e.g., luminescent strain and wildtype as a control) for 8-12 h (e.g., overnight) on YPD plates at $37^{\circ} \mathrm{C}$. Afterward, take a loop of Candida cells; dissolve in $1 \mathrm{ml}$ of PBS and wash twice with $1 \mathrm{ml}$ of PBS. Count the cells (e.g., with a Bürker chamber or spectrophotometer) to prepare Candida cell suspensions in RPMI-1640 medium at an appropriate cell density (as mentioned below) for subsequent use in the specific experiment.

2. Inoculate a sterile black or white (see Note 8) 96 -well cell culture plate by adding $100 \mu$ per well of a $1 \times 10^{7}$ cells/ml or $1 \times 10^{6}$ cells $/ \mathrm{ml}$ respectively for a C. albicans or C. glabrata cell suspension in RPMI-1640. Allow the Candida cells to adhere to the bottom of the wells by incubating the plate for $90 \mathrm{~min}$ at $37^{\circ} \mathrm{C}$. Wash the wells twice with PBS and then add $200 \mu \mathrm{l}$ of fresh RPMI-1640 medium to each well. Incubate the plate again at $37{ }^{\circ} \mathrm{C}$ for the duration of the experiment to allow mature biofilm formation on the bottom of the wells (C. albicans and C. glabrata form mature biofilms within 24-48 h).

3. To grow biofilms inside polyurethane catheters, preincubate $1 \mathrm{~cm}$ catheter pieces overnight with $\mathrm{FBS}$ at $37^{\circ} \mathrm{C}$. Place each catheter fragment in a clean 24-well plate (one catheter piece per well) and add $1 \mathrm{ml}$ of a Candida cell suspension $\left(5 \times 10^{4}\right.$ cells $/ \mathrm{ml}$ for $\mathrm{C}$. albicans or $1 \times 10^{6} \mathrm{cells} / \mathrm{ml}$ for $\mathrm{C}$. glabrata). Make sure that catheters are submerged in the medium and not floating on the top. Incubate the plate for $90 \mathrm{~min}$ at $37^{\circ} \mathrm{C}$ to allow cell attachment onto the substrate. Wash the catheters twice with $1 \mathrm{ml}$ PBS, transfer them to a clean plate, submerge them in fresh RPMI-1640 medium and incubate at $37^{\circ} \mathrm{C}$ for the duration of the experiment.

\subsubsection{BLI of In Vitro Biofilm Formation}

1. Freshly prepare the substrate solution (see materials: CTZ or D-luciferin) for in vitro experiments and initialize the BLI camera (see Note 9).

2. Take the culture plates containing biofilms (formed on the bottom of 96- or 24-well polystyrene cell culture plates or biofilms formed inside catheter pieces) out of the incubator.

3. Pipette the culture medium off and wash the biofilms twice with $1 \mathrm{ml}$ of sterile PBS (see Note 10), end by taking out all the PBS from the wells.

4. Insert the plate in the camera, set the appropriate field of view (FOV) and find the right imaging position for the plate by taking photographs. Set the settings for acquisition of the BLI image. Typically, we acquired BLI images with a 5-30 s exposure time at medium binning, depending on the signal.

5. Add swiftly but carefully (pipette under a $45^{\circ}$ against the well wall) the substrate working solution to the wells (100 $\mu \mathrm{l} /$ well substrate for 96 -well plates, $500 \mu \mathrm{l} /$ well CTZ or $700 \mu \mathrm{l} /$ well D-luciferin for 24-well plates), preferably with a 
multichannel pipette (see Note 11).

6. Immediately thereafter, insert the plates in the BLI-camera and start acquisition of consecutive frames until maximum signal is reached (see Note 12). Save the acquisitions that you need.

7. Quantify the BLI signal using the processing software of your BLI camera and report as photon flux per second (p/s) for a given region of interest (ROI) with a fixed size, covering the well or catheter (Fig. 1a, b).

Fig. 1

Regions of interest (ROIs) for BLI signal analysis. (a) for the analysis of the BLI signal from biofilms formed on the bottom of wells of a 96-well culture plate, a $8 \times 12$ grid can be used to quantify the photon flux from each well separately. (b) a rectangular ROI is placed over the catheter piece. (c) a rectangular ROI is placed over each catheter trio. (Reprinted from [24] with permission from Springer (C) 2014)

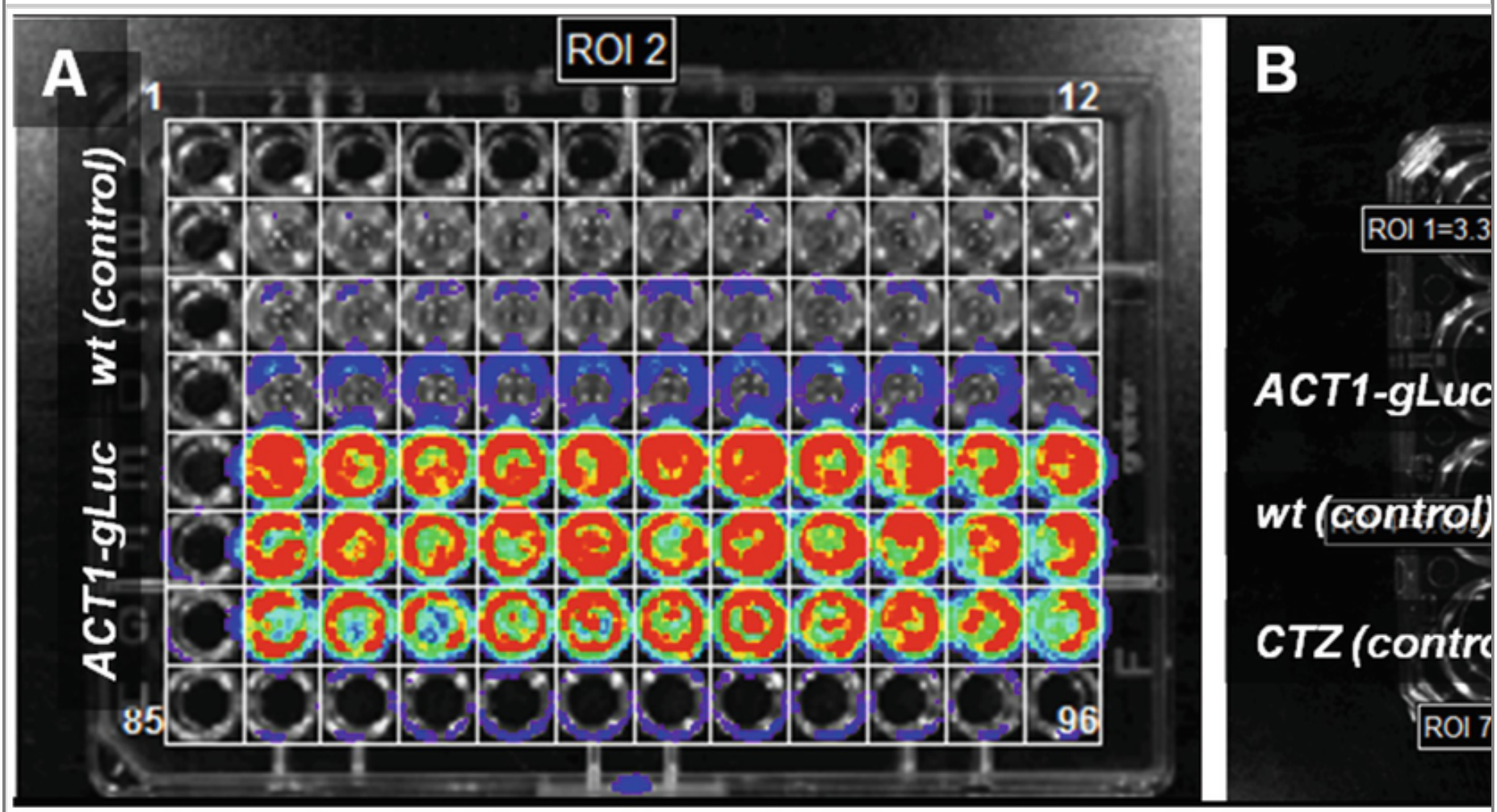

\subsubsection{In Vitro Screening of Antifungal Activity}

1. After $24 \mathrm{~h}$, when mature biofilms are formed, antifungal treatment can be initiated.

2. Add twofold serial dilutions of the antifungal drugs or vehicle (control) to the wells to cover a concentration range of $0.063 \mathrm{mg} / \mathrm{l}$ to $64 \mathrm{mg} / \mathrm{l}$.

3. Incubate the plates further for 24 or $48 \mathrm{~h}$ at $37^{\circ} \mathrm{C}$ before quantification by BLI.

\subsection{Bioluminescence Imaging of In Vivo Catheter-Associated Biofilms}

\subsubsection{In Vivo Biofilm Formation on Implanted Catheters}

1. Incubate the catheter pieces in $\mathrm{FBS}(100 \%)$ at $37^{\circ} \mathrm{C}$ overnight.

2. Place the serum-coated catheters into separate microcentrifuge tubes and add $1 \mathrm{ml}$ of the Candida cell suspension $\left(5 \times 10^{4}\right.$ cells $/ \mathrm{ml}$ for $\mathrm{C}$. albicans and $1 \times 10^{6}$ cells $/ \mathrm{ml}$ for $\mathrm{C}$. glabrata prepared in RPMI-1640).

3. Incubate for $90 \mathrm{~min}$ at $37^{\circ} \mathrm{C}$ for adhesion of the cells to the catheters.

4. After the adhesion period, place catheters on ice as fast as possible. 
5. Wash the catheters twice with $1 \mathrm{ml} \mathrm{PBS,} \mathrm{transfer} \mathrm{them} \mathrm{individually} \mathrm{into} \mathrm{clean,} \mathrm{sterile} \mathrm{microcentrifuge} \mathrm{tubes} \mathrm{and} \mathrm{place} \mathrm{on}$ ice for transport to the surgery room for implantation.

6. Prepare the animal for surgery. Anesthetize the animal by i.p. injection of ketamine-domitor anesthetic cocktail, wait a few minutes until the animal is fully asleep (check by pinching the tail or a paw: the animal should not react to that). Apply ophthalmic ointment on the eyes, shave the lower back of the animal, apply some xylocaine gel on the skin for local anesthesia of the area where you will implant the catheters (wait for a minute to work), disinfect the skin with iodine-isopropanol.

7. Make a small incision in the skin (approximately $0.5-1 \mathrm{~cm}$ ), carefully dissect the subcutis with a scissor to create two (for mice) or three (for rats) subcutaneous tunnels, make each tunnel about $1.5 \mathrm{~cm}$ long and $1 \mathrm{~cm}$ wide (Fig. 2).

8. Insert three catheter pieces (inoculated with the same Candida strain) in each tunnel, taking care that they fit next to each other in a horizontal arrangement and that they do not lay over each other. This way, you can implant six (for mice) or nine (for rats) catheter fragments in total and test biofilms formed by different Candida strains in the same animal (e.g., WT control strain and the corresponding bioluminescent strain).

9. Close the incisions by sutures and disinfect the wounds.

10. Wake the animal up by administering antisedan i.p. to reverse anesthesia (see Note 13).

Fig. 2

Catheter implantation. Start by making two incisions in the skin (full black lines in panel a). Next, by inserting scissors under the skin through these incisions, dissect the subcutaneous space to create two tunnels of about $1.5 \mathrm{~cm}$ long and $1 \mathrm{~cm}$ wide (dashed lines in a). Make sure that the tunnels do not make contact with each other. Through the incision, insert three catheters next to each other in each subcutaneous space (b). Close the incisions in the skin by separate surgical sutures (c). Pay attention that you place the sutures well under the catheters and not on top, in order not to block any of the emerging bioluminescence. (Reprinted from [24] with permission from Springer (C) 2014)

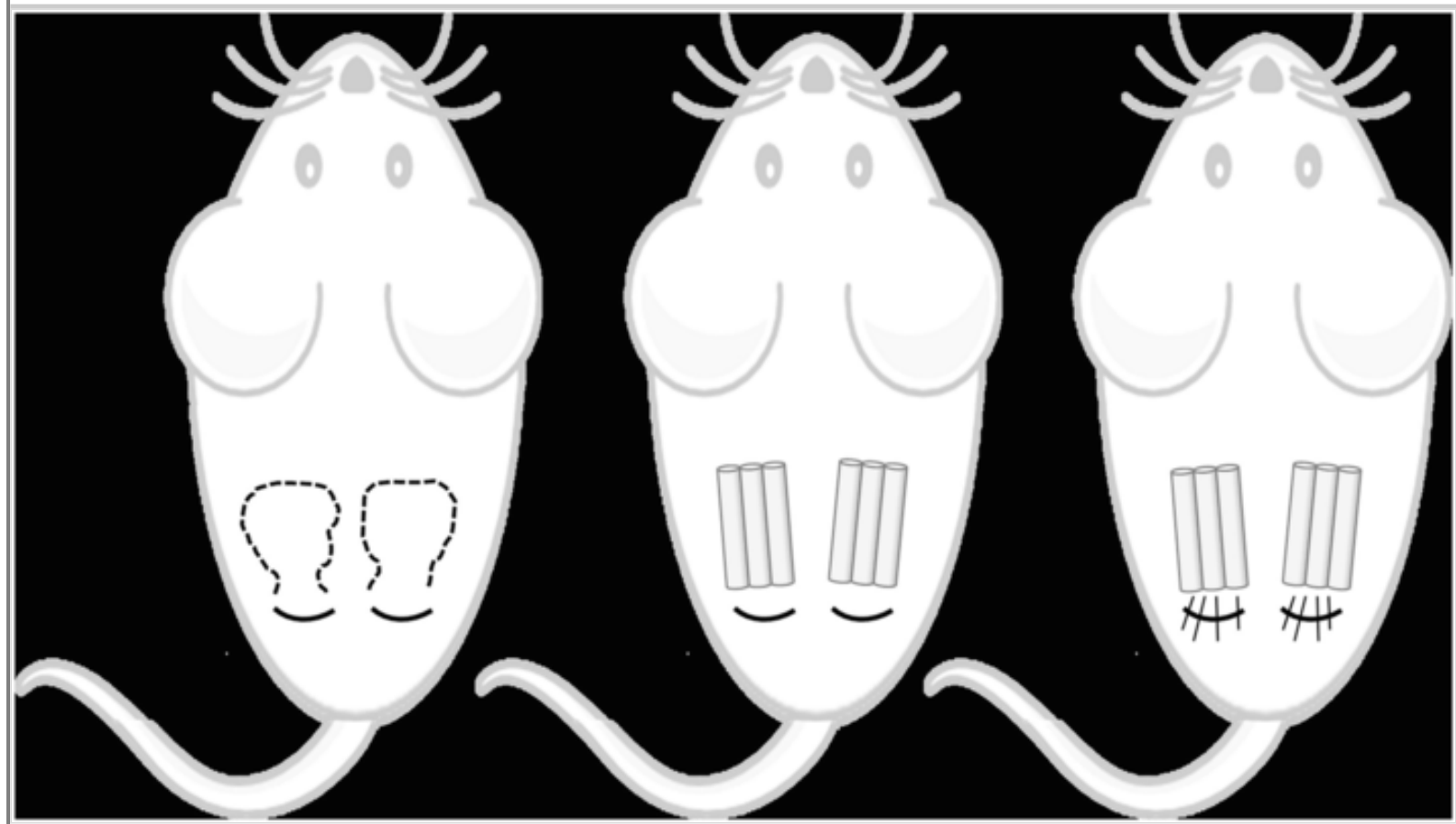

\subsubsection{BLI of In Vivo Biofilm Formation}

1. After infection with $\mathrm{C}$. albicans, the animals are typically imaged at baseline (day 0 , after catheter implantation), on day 2 when a mature biofilm is formed, and on day 6 when a mature biofilm is sustained. For C. glabrata, animals are imaged at baseline and continued on day 1,5 and 7.

2. In vivo BLI: Prepare fresh in vivo substrate working solution (see materials: CTZ or D-luciferin) just before the start of the BLI session and initialize the BLI camera (see Note 9). 
3. Anesthetize the animals using an induction box (comes usually with the BLI camera). Use a gas mixture of isoflurane in oxygen, $\mathrm{N}_{2} \mathrm{O} / \mathrm{O}_{2}$ or air, at $2-3 \%$ for mice and $3-4 \%$ for rats. Rats are best anesthetized and imaged one by one; mice can be put asleep by maximum of four at a time in the induction box and then sequentially imaged.

4. After induction, maintain anesthesia in the induction box and in the imaging chamber at $1.5-2 \%$ for mice and $2-3 \%$ for rats, at a flow rate of the carrier gas of $0.5 \mathrm{l} / \mathrm{min}$.

5. Before starting the imaging session, put the imaging plate in position $\mathrm{A}$, which corresponds to a FOV of $10 \mathrm{~cm}$. Reassure yourself of the right position of the anesthesia outlets and animal by placing a sleeping animal in the box and taking some photographs to confirm the correct imaging position, in the FOV right under the camera.

6. Prepare two syringes containing each $100 \mu \mathrm{l}$ of the substrate solution. Take one animal and use a nose cone with gas anesthesia to keep it asleep on the bench during the injection. Bring the needles of the syringes one by one in place subcutaneously above the catheters as depicted in Fig. 3 (see Notes 14-16). Use both hands to inject the substrate simultaneously on top of the catheters (see Note 17).

7. Immediately after injection, place the animal on the warm plate in the camera box and start the image acquisition. A typical BLI image is depicted in Fig. 4. Acquire consecutive scans with acquisition times ranging from 20 to $60 \mathrm{~s}$ (depending on the signal intensity) until the maximum signal intensity is reached (see Note 18). During the acquisition of the next frame, you can measure the BLI signal of the previously acquired frames by placing a ROI over each catheters trio and measuring the signal intensity (Fig. 1c).

8. Repeat from step 3 for the next animal(s).

9. Analyze the BLI data and report the BLI signal intensity of each catheter trio as photon flux per second for a rectangular ROI of fixed size placed over each catheter trio.

Fig. 3

Topical substrate injection for in vivo BLI. Insert the syringes in such a way that the largest part of the needle is subcutaneously (dashed lines) to avoid leakage of substrate out of the needle entrance point. Apply the substrate on top of the catheter trio (end of dashed line) simultaneously. (Reprinted from [24] with permission from Springer (C) 2014) 


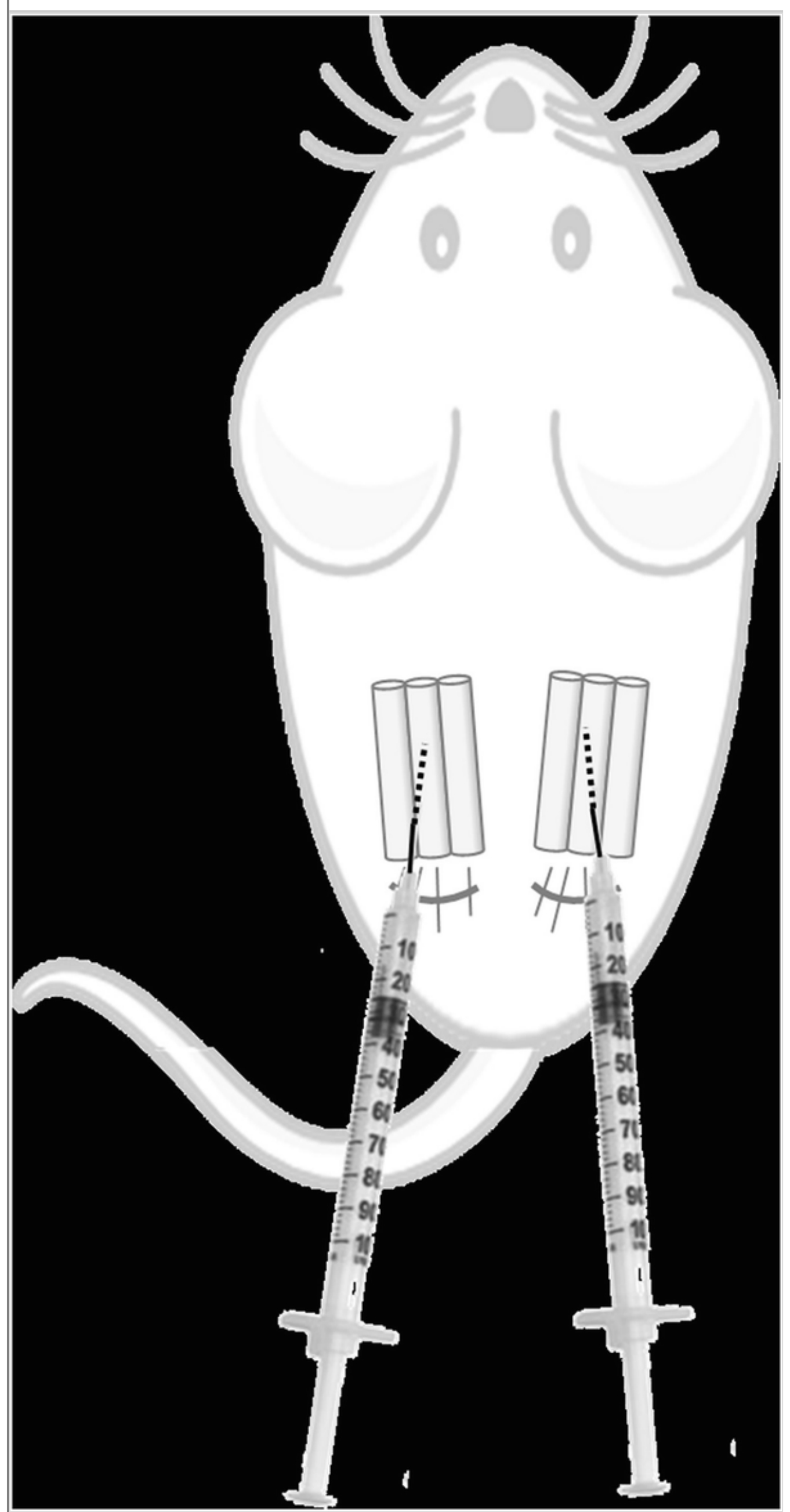


Fig. 4

Typical BLI image from a 2-day-old mature biofilm formed inside 3 catheters implanted left and right under the skin of a mouse. Left: biofilms formed by wild-type C. albicans SC5314, right biofilms formed by C. albicans SC5314 expressing gLUC. (Adapted from [24] with permission from Springer (C) 2014)

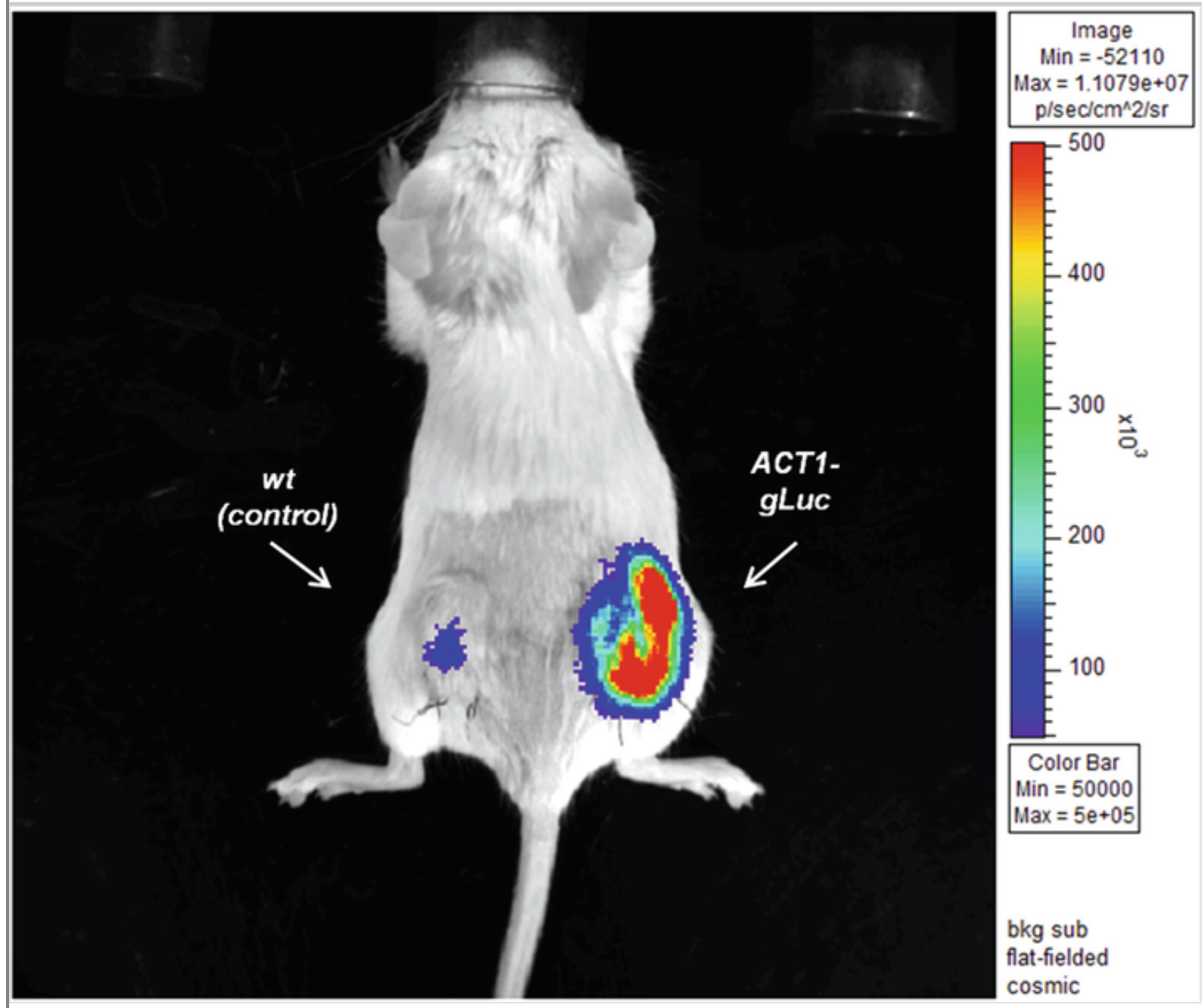

\subsubsection{In Vivo Screening of Antifungal Activity}

1. After 2 days of in vivo biofilm formation on the implanted catheters, use BLI to assess the baseline of mature biofilm fungal burden (see Subheading 3.2.2).

Start antifungal treatment and continue the treatment for 7 consecutive days by intravenous or i.p. injection of the antifungal drug or vehicle as a control. A typical BLI image of the result after treatment is depicted in Fig. 5. Appropriate concentrations of the antifungal drug should be determined during the in vitro screening of the antifungal activity.

\section{Fig. 5}

Typical BLI image from a 9-day old mature biofilm formed inside implanted catheters, and treated with or without caspofungin. In both mice, biofilms were formed by C. albicans expressing gLUC on 3 catheters implanted left and right. Mice were treated for 1 week with caspofungin or vehicle as a control and imaged thereafter. (Adapted from [21] with permission from Elsevier (C) 2018) 


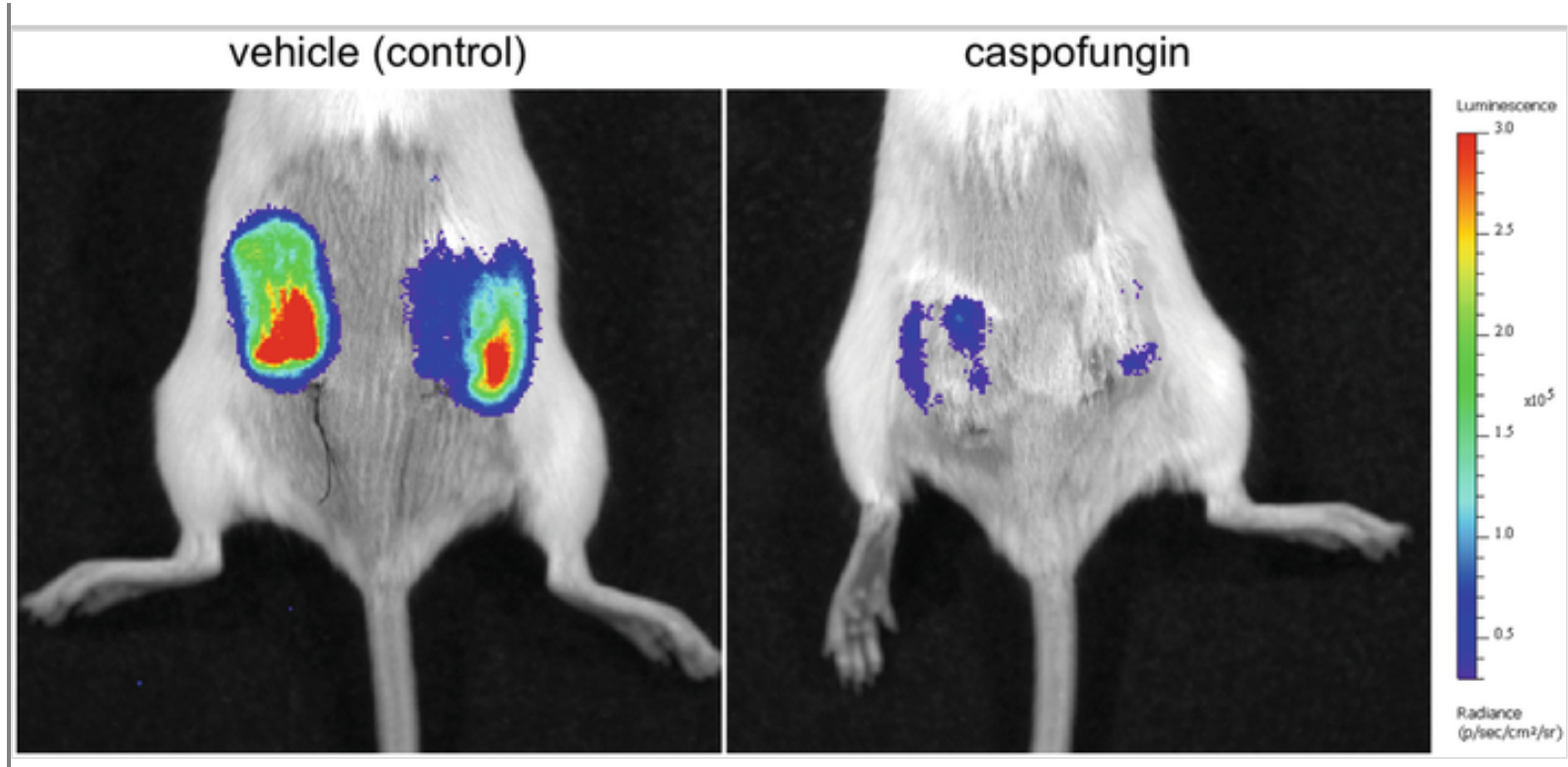

\subsubsection{Explantation of Catheters for Ex Vivo Biofilm Evaluation}

After the final imaging time point, recover the catheter pieces from the animals for further ex vivo analysis of the biofilms.

1. Prepare microcentrifuge tubes with $1 \mathrm{ml}$ PBS (each catheter will go into a separate microcentrifuge tube) and label them beforehand.

2. Euthanize the animals by cervical dislocation.

3. Disinfect the skin of the back with $0.5 \%$ chlorhexidine in $70 \%$ alcohol.

4. Make two new incisions above the catheters and remove the catheter fragments one by one from under the subcutaneous tissue using sterile tweezers (see Note 19).

5. Wash the catheter twice with $1 \mathrm{ml}$ sterile PBS and put it in the microcentrifuge tube (prepared in step 1).

6. Keep the tubes with catheters on ice or in a $4{ }^{\circ} \mathrm{C}$ refrigerator for short storage before further processing (i.e., quantification of biomass or with microscopy techniques).

7. Fungal burden quantification: Wash the cell culture plates, in vitro evaluated catheters or explanted catheters twice with $1 \mathrm{ml} \mathrm{PBS}$, sonicate for $10 \mathrm{~min}$ at $40,000 \mathrm{~Hz}$ in a water bath sonicator (Branson 2210) and vigorously vortex for $30 \mathrm{~s}$. Make 1:10 and 1:100 dilutions and plate $100 \mu \mathrm{l}$ of the original samples, 1:10 and 1:100 dilutions on YPD agar plates in duplicate. Incubate the plates for 2 days at $37^{\circ} \mathrm{C}$, after which you can count colony forming units (CFUs). Recalculate to fungal load per fragment (CFUs/catheter piece) taking into account the dilution factor and the plated volume.

8. Microscopical evaluation: To examine the three-dimensional structure of the biofilms formed on the inside of the explanted catheters, you can consider performing fluorescence microscopy, confocal laser scanning microscopy or scanning electron microscopy. For these procedures, we refer to [13].

\section{Notes}

1. Different materials may have different properties for attachment of fungal cells and subsequent biofilm development.

2. Intravenous catheters are often made radiopaque for ease of detection with X-ray imaging, once they are brought into place in the body. The substances added to make catheters radiopaque can elicit phosphorescent properties. We found the majority of catheters commonly used in hospitals to be strongly phosphorescent, which confounds BLI results.

Therefore, in our study we used the Certofix catheter from BBraun because it is not phosphorescent. If you would use another type of catheter, carefully check for phosphorescence by imaging it in the BLI camera at regular intervals, and check for an increase in signal after exposing the catheters to light, for example, by opening the scanner door and resuming BLI scanning.

3. We use Balb/C mice as this is a common background strain for many transgenic mouse models. For BLI, it is important that they are white (or nude) as the pigmentation of the skin, even when shaving the animals, absorbs a substantial amount of the BLI signal. 
4. The biofilms will also develop without immune suppression of the host, but the fungal load on the catheters may vary substantially in rats. When suppressing the immune system of rats, the fungal load recovered from the catheters is much more consistent [22], reducing the standard deviations and hence reducing the amount of animals needed to reach a given power in your experiment. Therefore, we recommend you to use the protocol for immune suppression before you start the catheter implantation in rats. For mice, we did not observe a similar reduction in variation of CFU counts when modulating the immune suppression [21].

5. For measuring luminescence in vitro, you can also use any luminometer or essentially any plate reader that is suitable for measuring luminescence. Some can even be equipped with injectors for controlled addition of the substrate. The advantage of placing the plates in the BLI system is that a luminometer/plate reader will not provide an image of your plate.

6. The IVIS systems come with a very comprehensive manual, it is worth reading through (the first part) of the manual and you will be able to get started with the camera and the software easily.

7. When diluting the stock solution to make the in vivo CTZ working solution, a small precipitate will form. According to the manufacturer this should not happen, but in our hands using the prescribed dilution protocol, we could never avoid having some extent of precipitation. As far as we know, other users experienced the same problem. It is imperative to dilute the CTZ immediately prior to injection as this will limit the precipitation.

8. For BLI measurements, it is recommended to use black or white cell culture plates, with or without transparent bottom. When not using these black or white plates, there will be BLI signal scattering from adjacent wells into the well you are measuring in case you have high signal intensities. In case you do not have a black or white plate at hand, you can also use a transparent plate and poor black drawing ink in between wells to reduce this "cross talk." This is not ideal as there will still be some "cross talk" where the wells are touching each other, as it will have some influence on the quantification of your BLI results. After your BLI experiment is finished, you can use a pipette to take out the ink for later reuse.

9. Starting up BLI camera: Before you start imaging, you need to login and initialize the system (button: "initialize"). If your BLI system is only used sporadically, it can be kept off in between imaging sessions. In that case, it will need to cool down before you can start imaging, which can take up to several hours depending on the system. In most cases, however, the system is left on to keep the CCD camera (partially) cooled and to take background data overnight. Initializing the system takes then only few minutes and you will be able to start almost immediately with your imaging experiment.

10. Especially when you are dealing with biofilms formed on the bottom of wells, take care not to detach the biofilm from the bottom of the plates while washing.

11. When doing in vitro BLI measurements, you could consider making fresh substrate and then incubate it in the dark for $\sim 30$ min before you add it to the wells. This way, the difference in auto-oxidation of the substrate solution (very relevant in the case of CTZ) will be minimal in case you cannot add the substrate to all the wells simultaneously. When you set up your experiments, always take nonluminescent wild type-controls and control wells where you only add substrate along in your analysis for comparison with unspecific background signal. On the other hand, if you are interested in measuring the signal kinetics, make sure you add the substrate "as simultaneously as you can" (when pipetting with a multichannel pipette, make sure that in every pipetting move you take along appropriate controls) and start image acquisition as fast as you can after substrate addition.

12. In practice, you will almost immediately reach the maximum signal when performing in vitro BLI measurements as diffusion of the substrate into the biofilms is fast, after which the signal will steadily decrease. Depending on your research question, you can take about three to four consecutive frames at maximum signal with the settings of your choice or keep acquiring short frames during a certain period if you are interested in signal kinetics.

13. We do not recommend keeping the animal under ketamine/domitor anesthesia and proceed to BLI immediately after surgery while it is still anesthetized. We recommend letting the animal completely recover from the injection anesthesia before starting isoflurane anesthesia for the imaging session. The latter procedure was well tolerated by the animals.

14. We evaluated signal kinetics in vivo upon different administration routes of the CTZ (intravenous, intraperitoneal, subcutaneous) and compared the BLI signal. We found that CTZ does not have a favorable biodistribution upon i.v. or i.p. injection. For this reason, we recommend to apply CTZ topically. Here, we applied D-luciferin topically in analogy to CTZ administration, but we recommend to always optimize the administration route for your own in vivo model system.

15. Upon substrate injection, it is crucial that no substrate leaks out of the little hole you make in the skin upon inserting the syringe. That is why it is crucial to keep $\sim 1 \mathrm{~cm}$ of the needle under the skin when you inject, as shown in Fig. 3. CTZ shows some extent of auto-oxidation. Therefore, any "leaked" CTZ on the skin of the animal would give rise to unspecific BLI signal that would significantly influence the BLI measurement. 
16. Injecting the substrate subcutaneously is obvious at the first time-point, as there is a lot of subcutaneous space because of the tunnels created during surgery. However, this will heal over time and there will be fibrous tissue formed around the catheters, making injecting subcutaneously less evident, especially when doing BLI at later time points (6-9 days after catheter implantation). Take care not to inject intracutaneously. In this case, you will notice immediately by a "bobble" appearing due to the intradermal substrate injection. No or only a very low signal will be seen.

17. In case experiments are performed on rats that have three (or more) tunnels with catheter trios, ask a colleague to assist you with injecting the substrate in all the tunnels simultaneously.

18. At the first time-points, the maximum signal is reached in less than $1 \mathrm{~min}$ after CTZ administration. For D-luciferin, the maximum signal is reached about 15 min after injection. The later after catheter implantation surgery, the longer it takes until this maximum signal is reached, presumably because of the increase of fibrous tissue around the catheters that makes the substrate diffuse more slowly toward the biofilms.

19. Make sure to decontaminate the tweezers in between catheter pieces having biofilms from different Candida strains.

\section{Acknowledgments}

This work was funded by the Fund for Scientific Research Flanders (FWO) Research community on biology and ecology of bacterial and fungal biofilms (FWO: WO.026.11N), FWO research grant G.0691.15N, and FWO PhD fellowship to KVD (FWO 1181818N) and KU Leuven IF grants STG/15/24 and C32/18/010. We thank Christophe d'Enfert for providing us with the Clp10::ACT1p-gLUC59 plasmid. All imaging experiments were conducted at the Molecular Small Animal Imaging Center (MoSAIC) core facility of the KU Leuven. (Parts of this protocol have been updated and reprinted from [24] by permission from Springer (C) 2014.)

\section{References}

1. Brown GD, Denning DW, Levitz SM (2012) Tackling human fungal infections. Science 336(6082):647. https://doi.org/10.112 6/science.1222236

2. Nucci M, Marr KA (2005) Emerging fungal diseases. Clin Infect Dis 41(4):521-526

3. Warnock DW (2006) Fungal diseases an evolving public health challenge. Med Mycol 44(8):697-705

4. Wisplinghoff H, Bischoff T, Tallent SM, Seifert H, Wenzel RP, Edmond MB (2004) Nosocomial bloodstream infections in US hospitals: analysis of 24,179 cases from a prospective nationwide surveillance study. Clin Infect Dis 39:309-317

5. Periroth J, Choi B, Spellberg B (2007) Nosocomial fungal infections: epidemiology, diagnosis, and treatment. Med Mycol 45(4):321-346

6. Eggimann P, Garbino J, Pittet D (2003) Epidemiology of Candida species infections in critically ill non-immunosuppressed patients. Lancet Infect Dis 3:685-702

7. Eggimann P, Garbino J, Pittet D (2003) Management of Candida species infections in critically ill patients. Lancet Infect Dis 3(12):772-785

8. Costa-Orlandi CB, Sardi JCO, Pitangui NS, de Oliveira HC, Sorzoni L, Galeane MC, Medina-Alarcón KP, Melo WCMA, Marcelino MY, Braz JD, Fusco-Almeida AM, Mendes-Giannini MJS (2017) Fungal biofilms and polymicrobial diseases. J Fungi (Basel) 3(2):E22

9. Nett J, Andes D (2006) Candida albicans biofilm development, modeling a host-pathogen interaction. Curr Opin Microbiol 9:340-345

10. Andes D, Nett J, Oschel P, Albrecht R, Marchillo K, Pitula A (2004) Development and characterization of an in vivo central venous catheter Candida albicans biofilm model. Infect Immun 72:6023-6031

11. Schinabeck MK, Long LA, Hossain MA, Chandra J, Mukherjee PK, Mohamed S, Ghannoum MA (2004) Rabbit model of Candida albicans biofilm infection: liposomal amphotericin B antifungal lock therapy. Antimicrob Agents Chemother 48:1727-1732

12. Lazzell AL, Chaturvedi AK, Pierce CG, Prasad D, Uppuluri P, Lopez-Ribot JL (2009) Treatment and prevention of Candida albicans biofilms with caspofungin in a novel central venous catheter murine model of candidiasis. J Antimicrob Chemother 64:567-570 
13. Řičicová M, Kucharíková S, Tournu H, Hendrix J, Bujdákova H, Van Eldere J, Lagrou K, Van Dijck P (2010) Candida albicans biofilm formation in a new in vivo rat model. Microbiol 156:909-919

14. Van Wijngaerden E, Peetermans WE, Vandersmissen J, Van Lierde S, Bobbaers H, Van Eldere J (1999) Foreign body infection: a new rat model for prophylaxis and treatment. J Antimicrob Chemother 44:669-674

15. Hutchens M, Luker GD (2007) Applications of bioluminescence imaging to the study of infectious diseases. Cell Microbiol 9:2315-2322

16. Dorsaz S, Coste AT, Sanglard D (2017) Red-shifted firefly luciferase optimized for Candida albicans in vivo bioluminescence imaging. Front Microbiol 8:1478

17. Enjalbert B, Rachini A, Vediyappan G, Pietrella D, Spaccapelo R, Vecchiarelli A, Brown AJ, d'Enfert C (2009) A multifunctional synthetic Gaussia princeps luciferase reporter for live imaging of Candida albicans infections. Infect Immun $77: 4847-4858$

18. Doyle TC, Nawotka KA, Kawahara CB, Francis KP, Contag PR (2006) Visualizing fungal infections in living mice using bioluminescent pathogenic Candida albicans strains transformed with the firefly luciferase gene. Microb Pathog 40:82-90

19. Papon N, Courdavault V, Lanoue A, Clastre M, Brock M (2014) Illuminating fungal infections with bioluminescence. PLoS Pathog 10(7):e1004179

20. Persyn A, Rogiers O, Brock M, Vande Velde G, Lamkanfi M, Jacobsen ID, Himmelreich U, Lagrou K, Van Dijck P, Kuchariková S (2019) Monitoring of fluconazole and caspofungin activity against in vivo Candida glabrata biofilms by bioluminescence imaging. Antimicrob Agents Chemother 63:e01555-18

21. Vande Velde G, Kuchariková S, Van Dijck P, Himmelreich U (2018) Bioluminescence imaging increases in vivo screening efficiency for antifungal activity against device-associated Candida albicans biofilms. Int J Antimicrob Agents 52:42-51

22. Kucharíková S, Tournu H, Holtappels M, Van Dijck P, Lagrou K (2010) In vivo efficacy of anidulafungin against Candida albicans mature biofilms in a novel rat model of catheter-associated candidiasis. Antimicrob Agents Chemother 54:44744478

23. Gillum AM, Tsay EYH, Kirsch DR (1984) Isolation of the Candida albicans gene for orotidine-5'-phosphate decarboxylase by complementation of S. cerevisiae ura3 and E. coli pyrF mutations. Mol Gen Genet 198:179-182

24. Vande Velde G, Kucharíková S, Van Dijck P, Himmelreich U (2014) Bioluminescence imaging of fungal biofilm development in live animals. Methods Mol Biol 1098:153-167 